\title{
Dynamical properties of two-dimensional Kerr cavity solitons
}

\author{
William J. Firth, Graeme K. Harkness, Angus Lord, and John M. McSloy \\ Department of Physics, University of Strathclyde, 107 Rottenrow, Glasgow G4 ONG, Scotland \\ Damià Gomila and Pere Colet \\ Instituto Mediterraneo de Estudios Avanzados, Consejo Superior de Investigacones Cientificas, Universitat Illes \\ Balears, Campus Universitat Illes Balears, E-07071 Palma de Mallorca, Spain
}

Received April 7, 2001; revised manuscript received August 24, 2001

\begin{abstract}
We present the results of our study of the dynamics of two-dimensional Kerr cavity solitons. The solitons are absolutely stable over a substantial parameter domain. We analyze their dynamics beyond the instability boundary, finding regions of stable oscillation and of fivefold or sixfold azimuthal instability. The Hopf oscillation is surprisingly robust, owing to the influence of a lower-amplitude unstable soliton. (C) 2002 Optical Society of America

OCIS codes: $190.0190,190.1450,190.3270,190.5530,190.4360,190.4420$.
\end{abstract}

\section{INTRODUCTION}

Many interesting spontaneous spatial structures have been predicted and observed in nonlinear optical systems. ${ }^{1}$ Among them are localized bright spots in

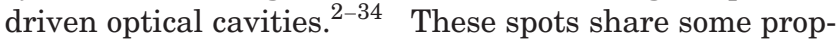
erties with spatial solitons and have become known as cavity solitons. Kerr cavity solitons are particularly fundamental and interesting in view of the properties of their propagating counterparts. Whereas in one transverse dimension Kerr spatial solitons are stable, it is well known that their two-dimensional (2D) counterparts are unstable against self-focusing collapse. ${ }^{35,36}$ The stability and dynamics of 2D Kerr cavity solitons (KCSs) are thus of particular interest. Their existence and structure was documented some years ago, ${ }^{11}$ but only unstable KCSs were found, though with indications that they might become stabilized for smaller cavity detunings. This stabilization was subsequently confirmed in a brief paper, ${ }^{12}$ but few details were presented and stability against only cylindrically symmetric perturbations was established, so the question of the azimuthal stability of $2 \mathrm{D} \mathrm{KCSs}$ was left open.

In this paper we remedy that omission. We find that in part of the domain in which they are radially stable, $2 \mathrm{D}$ KCSs are in fact azimuthally unstable. The existence of a broad domain of absolute stability for moderately small cavity mistunings is, however, fully confirmed.

We establish these results by an essentially exact, if numerically based, method ${ }^{27}$ that enables us to provide a much more comprehensive dynamical picture of $2 \mathrm{D} \mathrm{KCSs}$ than was previously available. This method gives information on the nature and spatial structure of the internal modes of the KCSs that become undamped at the stability boundaries. We confirm our findings by using dynamical simulations with starting conditions governed by our calculated mode structures. We find that the character of the cylindrically symmetric instability is a Hopf bifurca- tion, resulting in a breathing oscillation of the KCSs. The amplitude of this oscillation increases with the background intensity. The oscillating soliton is rather robust, and we show that it neither collapses nor decays, even well above the Hopf threshold. Where azimuthal instability to a mode $\exp ( \pm i m \phi)$ dominates, it may be preferentially of index $m=5$ or $m=6$. In this case the resultant dynamics leads to formation of an expanding pattern, which for the $m=5$ instability maintains fivefold symmetry as it grows. In both cases the emerging pattern is dynamical; each spot oscillates with a locationdependent phase. ${ }^{37}$

\section{KERR CAVITY SOLITONS}

We describe KCSs by using the standard mean-field cavity model of Lugiato and Lefever, ${ }^{38}$ in which alternation of propagation about the cavity with coherent addition of the input field is replaced by a single partial differential equation with a driving term:

$$
i \frac{\partial E}{\partial t}+\nabla^{2} E+|E|^{2} E=i \varepsilon\left(-E-i \theta E+E_{\mathrm{in}}\right),
$$

where $\nabla^{2}=\partial^{2} / \partial x^{2}+\partial^{2} / \partial y^{2}$. The three terms on the right-hand side of this are perturbations of the nonlinear Schrödinger equation, all small if $\varepsilon$ is. The first is a linear loss $(\varepsilon>0)$; the last, the driving field $E_{\text {in }}$ that is needed to sustain $E$ against that loss. The middle term, in $\theta$, describes the cavity mistuning with regard to the driving field (which we use as the frequency reference). Time is scaled to the cavity response time. This model [Eq. (1)] is appropriate for high finesse and assumes that only one longitudinal mode is excited.

We now set $\varepsilon=1$, which is equivalent to a rescaling, and designate the resultant equation the LugiatoLefever equation (LLE) ${ }^{38}$ Its only known exact solutions 


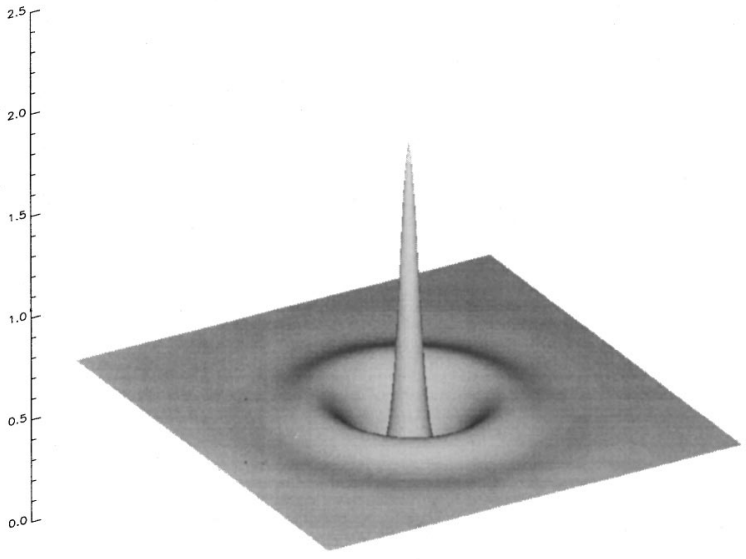

Fig. 1. Typical cavity soliton, showing a bright peak on a darker homogeneous background, with a few weak diffraction rings. Plotted is the modulus of the intracavity field as a function of the transverse coordinates $x$ and $y$; parameters are $\theta=1.2$ and $I$ $=0.9$.

assume a homogeneous (plane-wave) driving field and are themselves homogeneous, given by $E=E_{s}=E_{\text {in }} /[1$ $+i(\theta-I)]$, where $I=\left|E_{s}\right|^{2}$. For $\theta<\sqrt{3}$ this implicit solution is single valued (no optical bistability). 2D KCSs are usually rather unstable when $I$ is multiple valued, so we hence forth assume that $\theta<\sqrt{3}$ and can thus use intracavity background intensity $I$ and $\theta$ as convenient control parameters. Note that Lugiato and Lefever introduced the LLE in the context of pattern formation, finding that the plane-wave solution is always unstable to some transverse wave vector whenever $I \geqslant 1$. Stable KCSs can thus exist only for $I<1$, because they must asymptote to a stable $E_{s}$. Figure 1 shows a typical 2D KCS for $\theta=1.2$ and $I=0.9$. It consists of a bright peak on a flat background, with a few weak diffraction rings. The period and spatial damping rate of these rings follows from the fact that the KCS is asymptotically given by a pair of generalized Bessel functions. ${ }^{11,12}$ The rings become much more pronounced as $I \rightarrow 1$. For convenience, we often set $E=E_{s}(1+A)$, where $A(r)$ permits a background-free description of KCSs.

The LLE is known to have patterned solutions for $I$ $>1 .^{38}$ These include stripes (rolls), which are subcritical (i.e., coexist with a stable plane-wave solution) for $\theta$ $>41 / 30$. For smaller $\theta$, rolls exist only for $I>1$ and thus cannot coexist with stable 2D KCSs. Hexagonal patterns do, however, exist for $I<1, \theta<41 / 30$. Figure 2 shows a plot of $I$ versus the maximum $|A|$ for coexistent hexagons and 2D KCSs: $\theta=1.0$. These stationary solutions are calculated by use of a Newton method after the transverse derivatives have been computed with a Fourier transform. This approach is extremely accurate, and, furthermore, automatically generates the Jacobian operator whose eigenvalues determine the stability of the solution obtained.

For the cylindrically symmetric solutions we study stability with respect to perturbations of the form $\exp (\mathrm{im} \phi)$ for an arbitrary integer $m$, noting that for $m \neq 0$ closure of the linearized perturbation equations requires terms in both $m$ and $-m$. In this way we can study the stability of $2 \mathrm{D} \mathrm{KCSs}$ with respect to all possible perturbations in the $2 \mathrm{D}$ plane.

As might be expected, the negative-slope portions of both the KCS branch and the hexagon branch are unstable. The KCS branch usually has only a single undamped mode, as illustrated in Fig. 3. The positive-slope branch of KCSs is usually stable at low values of $I$ but becomes unstable as $I$ increases (Fig. 3). For $\theta=1.3$, a complex-conjugate pair of eigenvalues crosses the imaginary axis at $I \approx 0.79$, rendering the KCSs unstable. The underlying mechanism for this instability has been identified by Skryabin. ${ }^{39}$ Direct simulation confirms the stability analysis. A perturbed cavity soliton exhibits

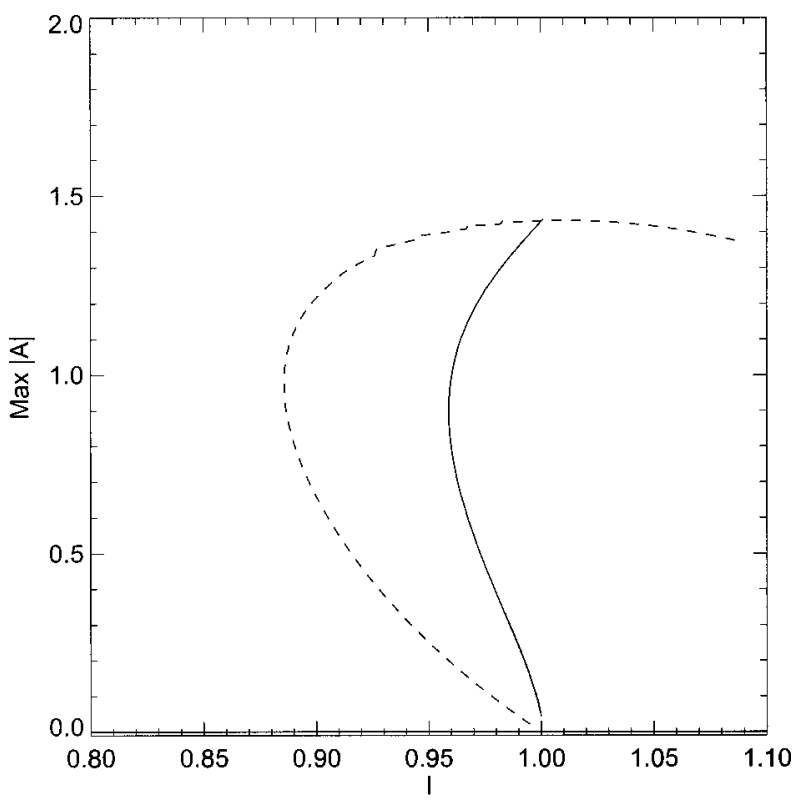

Fig. 2. Maximum amplitude of stationary solutions versus $I$ for $\theta=1.0$. Solutions shown are the 2D KCS solutions (solid curve) and the hexagonal pattern (dashed curve). The slight irregularity in the hexagon curve is a numerical artifact associated with the existence of a continuum of hexagonal patterns with different lattice spacings.

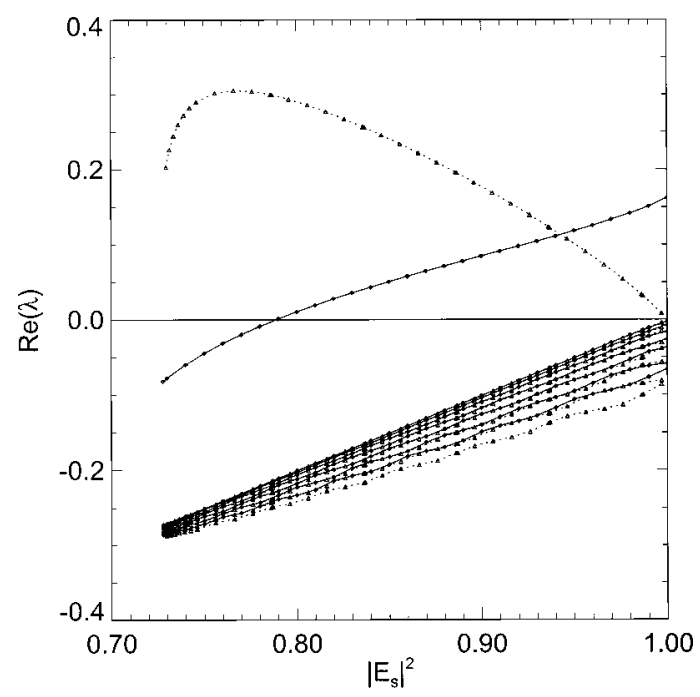

Fig. 3. Stability of the upper- (solid curves) and lower- (dashed curves) branch cavity solitons: how the eigenvalues with largest real parts change with $I \equiv\left|E_{s}\right|^{2}$. The detuning is $\theta=1.3$. 


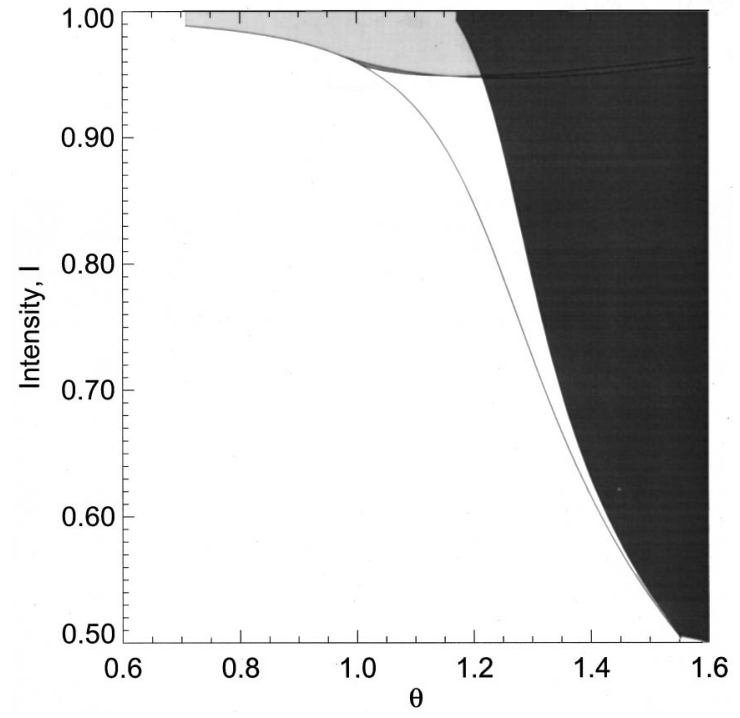

Fig. 4. Stability of $2 \mathrm{D}$ KCSs in the $\theta, I$ plane. Solitons exist above the lowest of the curves and are stable in the unshaded region. In the darkest area they are unstable to a Hopf mode with $m=0$, whereas in the other two shaded regions they are unstable to $m=5$ and $m=6$ modes (see text).

damped oscillations in the stable domain, which become undamped as the stability boundary is crossed. The positive-slope part of the hexagon branch can also be unstable to a Hopf mode. ${ }^{37}$ Hopf instability and the oscillation of a dark cavity soliton were previously reported and analyzed in a cavity with saturating defocusing nonlinearity. ${ }^{40}$

The stability of $2 \mathrm{D}$ KCSs for general $(\theta, I)$ is displayed in Fig. 4. The area above the lowest curve in Fig. 4 shows where 2D KCSs exist, and the shaded regions show where they are unstable. They exist only for a finite range $I_{\min }<I<1$, which is broader for large $\theta$. They are stable against $m=0$ perturbations only over a range $I_{\min }<I<I_{\text {hopf }}$ owing to the presence of a Hopf bifurcation in the dark shaded area. The Hopf-stable strip is very narrow for $\theta>1.5$, which is why no stable $2 \mathrm{D}$ KCSs were found in an earlier study. ${ }^{11}$

Figure 5 shows the results of a numerical integration of the LLE inside the Hopf-unstable region $(\theta=1.3, I$ $=0.9$ ). The initial condition was the unstable solution found by use of the Newton method. The exponential, oscillatory divergence is clear. In fact, the projection of the dynamics onto the Hopf eigenmode of the initial soliton shows an exponential growth, in excellent agreement with the computed positive eigenvalue. In Fig. 6 a cross section of the oscillatory solution is shown as a function of time. ${ }^{41}$

Figure 7 shows phase plots of the upper- and lowerbranch solitons together with the trace of the Hopf oscillation of its central peak. The full phase profiles over all $r$ are also plotted for the extrema. Clearly, at the lower extremum of its oscillation, the soliton dwells close to the lower-branch KCS. ${ }^{41}$

Even at $I=0.8$, only just beyond the Hopf threshold, the oscillating soliton's profile comes close, in both amplitude and phase, to that of the lower-branch KCS. It thus seems to act as a barrier, rather than a catalyst, to decay of the Hopf-unstable KCSs. We can explain this somewhat surprising phenomenon by considering the dynamics of the LLE in the vicinity of the lower branch KCS. From Fig. 3 it is evident that, for $I \sim 0.9$, this KCS has just one unstable mode, with all the others well damped. We can expand the total field as $E=E_{s}(1+A)=E_{s}(1$ $\left.+A_{l}+a A_{\text {unst }}+B\right)$, where $A_{l}$ is the lower-branch KCS and $A_{\text {unst }}$ is its unstable mode with amplitude $a$. Because there is only one unstable mode we can take $a$ to be real and such that, if $a>0$, the lower-branch soliton will evolve toward the upper branch. $B$ is the remainder of the deviation from the lower-branch KCS.

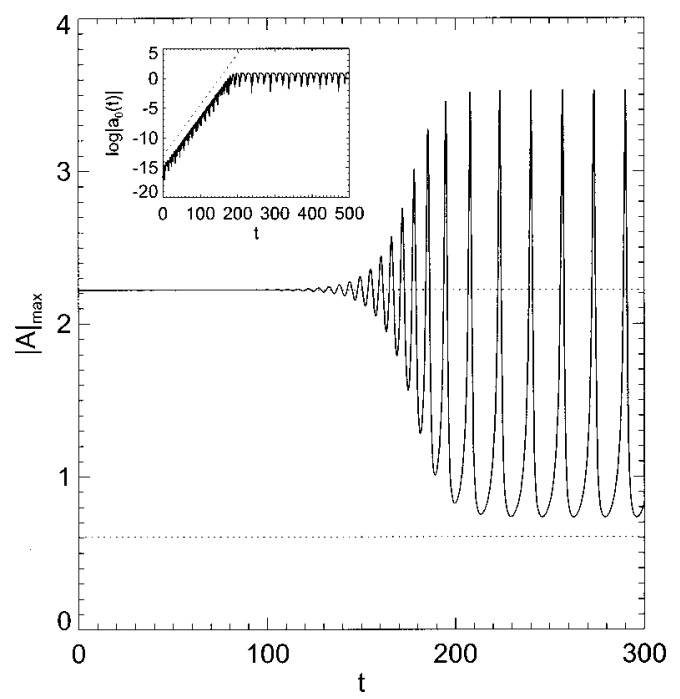

Fig. 5. Dynamics of oscillating 2D KCS beyond the Hopf bifurcation: $|A(0)|$, as a function of time $(\theta=1.3, I=0.9)$. Dashed lines show $|A(0)|$ for the upper- and lower-branch solitons. Inset, amplitude $\left|a_{0}\right|$ of Hopf-unstable eigenmode $v_{0}(\mathbf{r})$, where $A(\mathbf{r}, t) \approx A_{u}(\mathbf{r})+a_{0}(t) v_{0}(\mathbf{r}) ; A_{u}$ is the Hopf-unstable KCS and the dashed line is the gradient predicted by the corresponding eigenvalue from the linear stability analysis.

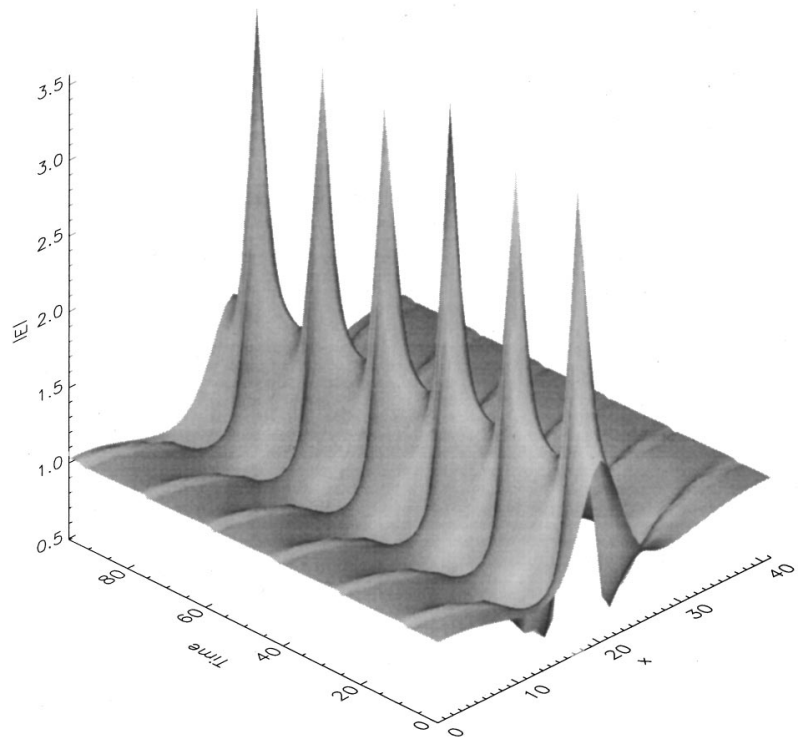

Fig. 6. Dynamics of an oscillating 2D KCS beyond the Hopf bifurcation $(\theta=1.3, I=0.9)$ : cross section $|E(x, y=0, t)|$ relative to $x$ and time. (The oscillation preserves cylindrical symmetry.) 


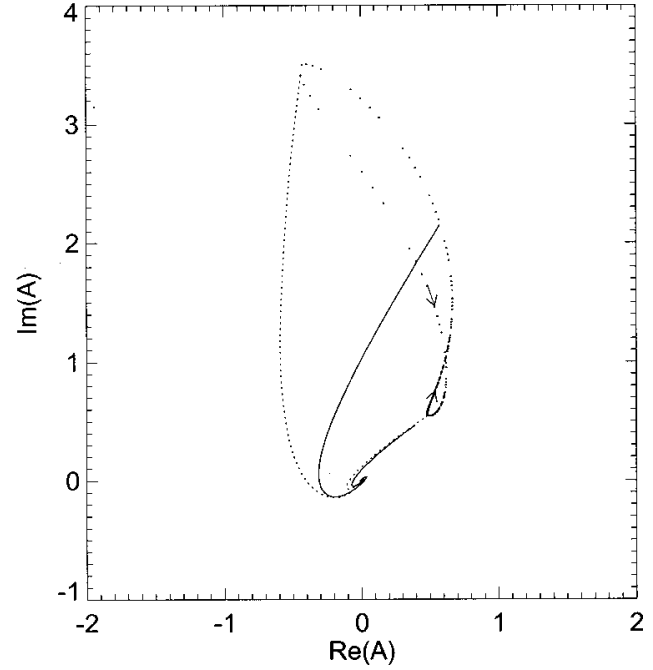

Fig. 7. Phase-plane representation of the dynamics of an oscillating $2 \mathrm{D} \operatorname{KCS}(\theta=1.3, I=0.9)$. Dashed curves, phase portrait of $A(r)$ at the extrema of the oscillation; dotted curves, $A(0)$ at intermediate times. Arrows, directions of rotation. Upper and lower solid curves (Hopf-unstable) upper and (amplitude unstable) lower KCSs, respectively.

Close enough to the unstable soliton $A_{l}$, we can linearize in $a$ and $B$. It follows from the linear stability properties of $A_{l}$ that $a$ grows exponentially, whereas $B$ decays at least as fast as the least-damped stable mode of $A_{l}$. Thus the dynamics rapidly becomes one dimensional, confined to the unstable manifold of the lower-branch KCS. The exception occurs when $a=0$, which asymptotes to $A_{l}$, because it has no projection along the unstable mode. The condition $a=0$ identifies the stable manifold of $A_{l}$. For the present case the unstable manifold is one dimensional in the function space of the eigenmodes of $A_{l}$ and the stable manifold is infinite dimensional. Because of the simple dynamics of $a$, its time dependence is $a(t)$ $=a(0) \exp (\lambda t)$, where $\lambda$ is the positive eigenvalue of $A_{l}$. It follows that (within the linear approximation) the stable manifold acts as an absolute barrier: Crossing it would require that $a$ change sign, which is impossible (unless some noise or other effect perturbs the LLE evolution). Physically, this structure implies that any bump in $A$ that is close enough to $A_{l}$ will evolve toward $A_{l}$ if $|a|$ is initially small enough compared with $|B|$. The exponential growth of $a$ eventually takes over, however, and the bump either decays into the background (if $a<0$ ) or evolves toward the upper-branch soliton (if $a>0$ ).

The oscillation of the Hopf-unstable KCS, by its nature, is such that if it should approach the stable manifold of the lower-branch soliton it will do so with $a>0$. If this $a$ is initially small compared with $|B|$, the soliton is attracted toward $A_{l}$ as $B$ decays and then escapes along the unstable manifold as the exponential growth of $a$ takes over. This procedure yields a prediction about the subsequent dynamics: Because the unstable manifold of the lower-branch KCS is also the stable manifold of the upper-branch KCS, the oscillating soliton should follow its close encounter with the former by a close encounter with the latter. That this is indeed what happens is evident from Fig. 7. This surprising robustness of the oscil- lating $2 \mathrm{D} \mathrm{KCS}$ is potentially significant in relation to applications because an oscillating soliton is almost as clearly distinguishable from no soliton, as is a stable soliton (because its amplitude is bounded below by that of the lower-branch soliton).

As we already mentioned, however, the barrier can be crossed in the presence of deviations from the deterministic evolution. Noise in experiments is unavoidable, which would undoubtedly limit the robustness in practice of Hopf-unstable cavity solitons. Even in simulations we have seen decay of the oscillating KCS, which we ascribe to numerical noise because increasing the computational accuracy prevents decay. It is likely that the decay seen in earlier research with $\mathrm{KCSs}^{11}$ is, with hindsight, attributable to numerical noise. It would be interesting to investigate whether the decay of the oscillating dark-cavity soliton reported by Michaelis et al. ${ }^{40}$ is also sensitive to numerics. Of course, our analysis of the soliton dynamics has only first-order validity and cannot establish whether the stable manifold is a global decay barrier.

The mechanism by which the unstable manifold of the lower-branch cavity soliton acts as a phase-space barrier seems quite general, so this phenomenon should not be limited to pure Kerr, or even Kerr-like, media. Recent studies with a semiconductor model ${ }^{33}$ identified the role of the lower-branch soliton as a separatrix, in that below a certain amplitude an address pulse failed to write a soliton, whereas above that critical amplitude the pulse successfully initiated one (which in the case in question was stable). Therefore a weak pulse creates a bump with $a$ $<0$, leading to decay, whereas the critical address pulse corresponds to $a=0$. Any stronger pulse generates a bump with $a>0$, which evolves into an upper-branch soliton. Such separatrix behavior in the writing of solitons was experimentally observed in a semiconductor cavity $^{20}$ and for cavity-soliton-like feedback structures. ${ }^{29,34}$ In the latter cases the critical switching dynamics was used to map out the unstable lower branch.

The dynamics close to the lower-branch soliton has similarities to dynamics close to a saddle point in lowdimensional dynamical systems. One characteristic of such dynamics is sensitivity to initial conditions and to noise. This sensitivity could account for the slight peakto-peak variation in Fig. 6. Whether this fluctuation is noisy or chaotic requires further, detailed, investigation.

For the approximate range $\theta<1.22$ shown in Fig. 4, the leading instability observed is not the Hopf mode (which is cylindrically symmetric) but instead modes with $m=5$ or $m=6$. This azimuthal instability leads to its surrounding ring's breaking up into five or six spots, respectively. Numerical integration shows that the resultant structure then grows to invade the homogeneous background. The leading instability is to $m=6$ for $\theta$ $<1.1$, leading to an (unstable) hexagonal pattern. For a narrow domain about $\theta=1.2$ (Fig. 4), $m=5$ dominates, and the resultant pattern, though it is locally hexagonal, retains its global fivefold symmetry (Fig. 8).

These azimuthal instabilities are easily interpreted as a quasi-1D modulational instability of the primary ring of the $2 \mathrm{D} \mathrm{KCS}$, once the local intensity $|E|^{2}$ on the ring exceeds unity by a sufficient margin to compensate for the ring's radial confinement. 


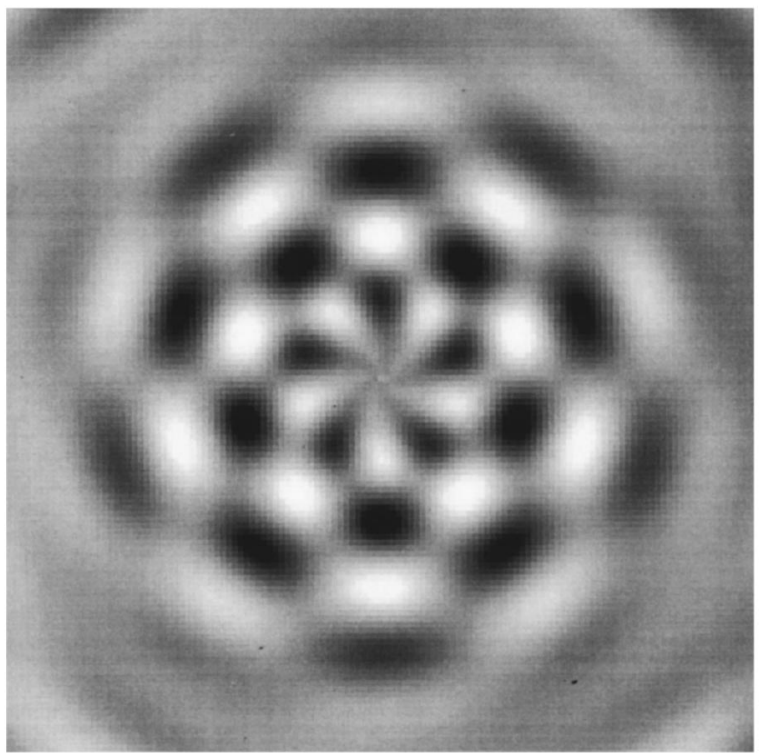

Fig. 8. Eigenmode corresponding to an azimuthal $m=5$ instability. $\theta=1.2, I=0.95$.

\section{SUMMARY}

We have demonstrated existence of stable 2D Kerr cavity solitons in the archetypal Lugiato-Lefever mean-field model. This is a behavior that is qualitatively different from its bulk-medium equivalent and so clearly demonstrates that cavity solitons are qualitatively different from ordinary spatial solitons. We have also presented detailed and accurate investigations of the nature of the processes by which this 2D Kerr cavity soliton may become unstable. We found a novel and interesting azimuthal instability, leading to formation of an unstable quasi-hexagonal pattern. There is an interesting instability of the Hopf type. We showed that the resultant dynamics preserves the soliton even when the oscillation reaches quite a high amplitude and have demonstrated the key role of the lower-branch soliton in this phenomenon.

\section{ACKNOWLEDGMENTS}

This study is partially supported by European Strategic Programme for R \& D in Information Technology project 28235, "Processing of Information by Arrays of Nonlinear Optical Solitons," and Engineering and Physical Sciences Research Council (UK) grants GR/M 19727 and GR/ N19830. We thank Tommaso Maggipinto, Andrew Scroggie, and Dmitry Skryabin for important contributions to the theory underlying this research.

W. J. Firth's e-mail address is willie@phys.strath.ac.uk.

\section{REFERENCES AND NOTES}

1. L. A. Lugiato, ed., Special issue on nonlinear optical systems, chaos, and noise, Chaos, Solitons Fractals 4, 12511258 (1994).

2. D. W. McLaughlin, J. V. Moloney, and A. C. Newell, "Solitary waves as fixed-points of infinite-dimensional maps in an optical bistable ring cavity," Phys. Rev. Lett. 51, 75-78 (1983).

3. J. V. Moloney and A. C. Newell, Nonlinear Optics (AddisonWesley, Redwood City, Calif., 1992).

4. N. N. Rosanov and G. V. Khodova, "Switching of a bistable interferometer on localized inhomogeneities," Opt. Spectrosc. (USSR) 65, 1399-1401 (1988).

5. N. N. Rosanov and G. V. Khodova, "Diffractive autosolitons in nonlinear interferometers," J. Opt. Soc. Am. B 7, 10571065 (1990).

6. G. S. McDonald and W. J. Firth, "Spatial solitary-wave optical memory," J. Opt. Soc. Am. B 7, 1328-1335 (1990).

7. S. Wabnitz, "Suppression of interactions in a phase-locked soliton optical memory," Opt. Lett. 18, 601-603 (1993).

8. G. S. McDonald and W. J. Firth, "Switching dynamics of spatial solitary wave pixels," J. Opt. Soc. Am. B 10, 10811089 (1993).

9. M. Tlidi, P. Mandel, and R. Lefever, "Localized structures and localized patterns in optical bistability," Phys. Rev. Lett. 73, 640-643 (1994).

10. W. J. Firth and A. J. Scroggie, "Optical bullet holes: robust controllable localized states of a nonlinear cavity," Phys. Rev. Lett. 76, 1623-1626 (1996).

11. W. J. Firth and A. Lord, "Two-dimensional solitons in a Kerr cavity," J. Mod. Opt. 43, 1071-1077 (1996).

12. W. J. Firth, A. Lord, and A. J. Scroggie, "Optical bullet holes," Phys. Scr. T67, 12-16 (1996).

13. M. Brambilla, L. A. Lugiato, and M. Stefani, "Interaction and control of optical localized structures," Europhys. Lett. 34, 109-114 (1996).

14. M. Tlidi and P. Mandel, "Spatial patterns in nascent optical bistability," Chaos, Solitons Fractals 4, 1475-1485 (1996).

15. A. J. Scroggie, W. J. Firth, G. S. McDonald, M. Tlidi, R. Lefever, and L. A. Lugiato, "Pattern formation in a passive Kerr cavity," Chaos, Solitons Fractals 4, 1323-1354 (1996).

16. S. Longhi, "Dark solitons in degenerate optical parametric oscillators," Opt. Lett. 21, 860-862 (1996).

17. G. Steinmeyer, A. Schwache, and F. Mitschke, "Quantitative characterization of turbulence in an optical experiment," Phys. Rev. E 53, 5399-5402 (1996).

18. M. Brambilla, L. A. Lugiato, F. Prati, L. Spinelli, and W. J. Firth, "Spatial soliton pixels in semiconductor devices," Phys. Rev. Lett. 79, 2042-2045 (1997).

19. L. Spinelli, G. Tissoni, M. Brambilla, F. Prati, and L. A. Lugiato, "Spatial solitons in semiconductor microcavities," Phys. Rev. A 58, 2542-2559 (1998).

20. V. B. Taranenko, I. Ganne, R. J. Kuszelewicz, and C. O. Weiss, "Patterns and localized structures in bistable semiconductor resonators," Phys. Rev. A 61, 0638-18 (2000).

21. C. Etrich, U. Peschel, and F. Lederer, "Solitary waves in quadratically nonlinear resonators," Phys. Rev. Lett. 79, 2454-2457 (1997).

22. D. Michaelis, U. Peschel, and F. Lederer, "Multistable localized structures and superlattices in semiconductor optical resonators," Phys. Rev. A 56, R3366-R3373 (1997).

23. K. Staliunas and V. J. Sanchez-Morillo, "Localized structures in degenerate parametric oscillators," Opt. Commun. 139, 306-312 (1997).

24. M. Tlidi, P. Mandel, and M. Haelterman, "Spatiotemporal patterns and localized structures in nonlinear optics," Phys. Rev. E 56, 6524-6530 (1997).

25. D. V. Skryabin and W. J. Firth, "Interaction of cavity solitons in degenerate optical parametric oscillators," Opt. Lett. 24, 1056-1058 (1999).

26. I. V. Barashenkov and E. V. Zelyanaya, "Stable complexes of parametrically driven, damped nonlinear Schrödinger solitons," Phys. Rev. Lett. 83, 2568-2571 (1999).

27. W. J. Firth and G. K. Harkness, "Cavity solitons," Asian J. Phys. 7, 665-677 (1998).

28. N. N. Rosanov, D. V. Fedorov, and G. V. Khodova, "Particlelike light structures in the wide-aperture laser with saturating absorption," J. Exp. Theor. Phys. 107, 376-392 (1995).

29. B. Schäpers, M. Feldmann, T. Ackemann, and W. Lange, "Interaction of localized structures in an optical patternforming system," Phys. Rev. Lett. 85, 748-751 (2000). 
30. W. J. Firth, "Processing information with arrays of spatial solitons," in Photonics Devices, and Systems, M. Hrabovsky, M. Miller, and P. Tomanek, eds., Proc. SPIE 4016, 388-394 (2000).

31. G. Tissoni, L. Spinelli, M. Brambilla, T. Maggipinto, I. Perrini, and L. A. Lugiato, "Cavity solitions in passive bulk semiconductor microcavities. I. Microscopic model and modulational instabilities," J. Opt. Soc. Am. B 16, 20832094 (1999).

32. G. Tissoni, L. Spinelli, M. Brambilla, T. Maggipinto, I. Perrini, and L. A. Lugiato, "Cavity solitons in passive bulk semiconductor microcavities. II. Dynamical properties and control," J. Opt. Soc. Am. B 16, 2095-2105 (1999).

33. T. Maggipinto, M. Brambilla, G. K. Harkness, and W. J. Firth, "Cavity solitons in semiconductor microresonators: existence, stability, and dynamical properties," Phys. Rev. E 62, 8726-8739 (2000).

34. A. Schreiber, B. Thüering, M. Kreuzer, and T. Tschudi, "Experimental investigation of solitary structures in a nonlin- ear optical feedback system," Opt. Commun. 136, 415-418 (1997).

35. N. N. Akhmediev and A. Ankiwicz, Solitons: Nonlinear Pulses and Beams (Chapman \& Hall, London, 1997).

36. A. C. Newell, Solitons in Mathematics \& Physics (Society for Industrial and Applied Mathematics, Philadelphia, Pa. 1987).

37. D. Gomila, M. Santagiustina, P. Colet, and M. San Miguel, "From hexagons to optical turbulence," preprint.

38. L. A. Lugiato and R. Lefever, "Spatial dissipative structures in passive optical systems," Phys. Rev. Lett. 58, 2209-2211 (1987).

39. D. V. Skryabin, "Energy of the soliton internal modes and broken symmetries in nonlinear optics," J. Opt. Soc. Am. B 19, 529-536 (2002)

40. D. Michaelis, U. Peschel, and F. Lederer, "Oscillating dark cavity solitons,” Opt. Lett. 23, 1814-1816 (1998).

41. A dynamical simulation may be viewed at http:// cnqo.phys.strath.ac.uk/movies/kerrhopf.mpg. 